Emerald Journal of Forensic Practice

\title{
Co-morbidity of personality disorder and clinical syndrome in high-risk incarcerated offenders
}

\begin{tabular}{|r|l|}
\hline Journal: & Journal of Forensic Practice \\
\hline Manuscript ID & JFP-05-2016-0026.R4 \\
\hline Manuscript Type: & Research Paper \\
\hline Keywords: & $\begin{array}{l}\text { personality disorder, clinical disorder, high risk, comorbidity, Axis I, Axis II, } \\
\text { offenders }\end{array}$ \\
\hline \multicolumn{2}{|l}{} \\
\hline
\end{tabular}

SCHOLARONE

Manuscripts 


\title{
Co-morbidity of personality disorder and clinical syndrome in high-risk incarcerated offenders
}

\begin{abstract}
Purpose: In light of the clinical importance of understanding co-morbidity within offender populations, this study examined the prevalence and comorbidities of clinical disorder (Axis I) and personality disorder (Axis II) within a sample of high risk, male offenders located in a high secure, prison-based personality disorder treatment service.
\end{abstract}

Methodology: The study utilised clinical assessment data for both Axis I diagnoses (Structured Clinical Interview for DSM-IV) and Axis II diagnoses (International Personality Disorder Examination) of personality disordered offenders $(n=115)$ who met the criteria for the treatment service between 2004 and 2015.

Findings: Co-morbidity between Axis I and Axis II diagnosis was high, with $81 \%$ of the sample having co-morbid personality disorder and clinical disorder diagnosis. The most prevalent Axis I disorder was substance misuse, and Axis II was anti-social, borderline, and paranoid personality disorder. Following Chi-square analysis, Cluster A personality disorder demonstrated co-morbidity with both mood disorder and schizophrenia/other psychotic disorder. Paranoid, schizoid, narcissistic, and avoidant personality disorder demonstrated a level of co-morbidity with Axis I disorders. There was no association found between the clinical disorders of substance use and anxiety with any personality disorder within this sample.

Value: In part these results suggest that certain Axis II disorders may increase the risk of lifetime Axis I disorders. The findings of no co-morbidity between the clinical disorders of substance use and anxiety with any personality disorder within sample is inconsistent to previous findings. 


\section{Introduction}

Recognition and understanding of co-morbidity is fundamental in the assessment of offenders (Blackburn, 2000); the presence of one disorder can affect the "treatment, course and phenomenology of another" (Tyrer, Gunderson, Lyons \& Tohen, 1997). The co-morbidity between Axis I and Axis II diagnosis within a forensic population has previously been examined in high secure hospitals (Coid, 2003; Blackburn, Logan, Donnelly \& Renwick, 2003), inpatient samples (Oldham et al., 1995), prisoner samples (Coid et al., 2009) and community based samples (Links \& Eynan, 2013).

The most prevalent findings in studies examining associations between Axis I and Axis II disorders is that a diagnosis of depression is significantly (and positively) associated with borderline personality disorder. This finding has been observed within varying forensic samples (Blackburn et al., 2003; Coid, 2003; Coid et al., 2009; Links \& Eynan, 2013; Oldham et al., 1995; Skodol et al., 2011). For example, $35 \%$ of a general prison population in England and Wales had diagnoses of borderline personality disorder and depression (Coid et al., 2009). Within high secure hospitals in England, this figure has been as high as $61 \%$ (Coid, 2003). A consistent association has also been observed between mood disorders and varying Axis II disorders (Oldham et al., 1995, Blackburn et al., 2003; Coid, 2003).

Relationships between schizophrenia and personality disorder also features within the literature (Coid, 2003; Coid et al., 2009). This includes co-morbidity with schizoid personality disorder in the general prison population (Coid, 2003), as well as co-morbidity with paranoid and schizotypal personality disorders within the general prison population (Coid, 2003) and the high secure psychiatric population within the UK (Coid et al., 2009). This suggests that Cluster A personality disorder diagnoses and schizophrenia co-exist to a significant level within forensic samples.

Substance use disorders have been identified as the most prevalent Axis I syndrome and having the highest co-morbidity with personality disorder within the UK prison population (Coid et al, 2009). A plethora of studies have identified co-morbidity between substance use disorder and antisocial personality disorder within UK forensic samples in the general prison population (Coid et al., 2009), and high secure settings (Blackburn et al., 2003; Coid, 2003). A diagnosis of borderline personality disorder has been shown to be related to a diagnosis of substance use disorder within UK prison populations (Coid et al., 2009) and high secure hospitals (Blackburn et al., 2003). These findings are predictable given that substance use behaviour is an identified trait within the criminal lifestyle aspect of antisocial personality disorder and the potentially self-damaging behaviours within borderline personality disorder. Research has identified substance use disorders as being co-morbid with paranoid, obsessive-compulsive and avoidant personality disorders within the UK prison population (Coid et al., 2009). Substance use has also been found to be comorbid with histrionic personality disorder within both inpatients and outpatients in the US (Oldham et al., 1995), and narcissistic personality disorder within a mainly community focussed review of US samples (Links \& Eynan, 2013).

More varied evidence exists for the presence of co-morbidity between anxiety disorder and various personality disorders across the literature base. This includes narcissistic, (Coid et al., 2009; Links \& Eynan, 2013); schizotypal (Coid et al., 2009; Blackburn et al., 2003); obsessive-compulsive (Coid et al., 2009); avoidant (Coid et al., 2009; Links \& Eynan, 2013; Blackburn et al., 2003); antisocial (Links \& Eynan, 2013; Hodgins et al., 2010), borderline, dependant (Links \& Eynan, 2013); and paranoid personality disorders (Blackburn et al., 2003). This indicates there is varying evidence for the presence (and absence) of co-morbidity between Axis II and anxiety related Axis I disorders within differing samples.

Scant evidence is present within the literature base relating to somatoform disorders' co-morbidity with personality disorder. Specifically, somatoform disorder has been identified as having co-morbidity with borderline personality disorder within UK high security psychiatric populations (Blackburn et al., 
2003; Coid, 2003). Outside of forensic psychiatric populations, somatoform disorders have been found to be co-morbid with borderline personality disorder in a sample of American personality disordered inpatients (Zanarini et al., 1998).

Whilst these findings have yielded valuable insight into the co-morbidity of forensic samples, it is imperative to acknowledge restrictions on the generalisbility of these findings, taking into account that co-morbidity is likely to vary according to the population in which it is studied. To the researchers' knowledge, there is no study to date that has exclusively explored the prevalence of co-morbid diagnoses within high-risk incarcerated offenders diagnosed with complex personality disorders. Therefore, it is concluded that there is an important gap in the literature must be explored. Increasing the understanding of co-morbidity within this specific population is pertinent at present. That is, the Offender Personality Disorder Pathway (Joseph \& Benefield, 2012) has been introduced in England and Wales with the intention of providing a progression pathway (across security levels) for offenders likely to be diagnosed with a personality disorder. This pathway was necessitated by approximately two thirds of offenders being considered to meet the criteria for at least one diagnosed personality disorder (Department of Health and Ministry of Justice, 2011).

The Offender Personality Disorder Pathway includes services within the high secure prison estate based at The Westgate Unit at HMP Frankland (which provides a treatment approach based on cognitive behavioural therapy; Bennett, 2015) and The Fens Unit at HMP Whitemoor (which has a cognitive interpersonal based treatment model; Saradjian et al., 2013). Criteria for meeting these services includes offenders who:

- pose a high risk of re-offending;

- have been diagnosed with complex personality disorder (which can include the presence of psychopathy); and

- have a functional link between their personality disorder diagnoses and their offending.

The discernible presence of clinical disorders and personality disorders can impact severely on treatment engagement for these high-risk complex individuals. These manifest in the form of treatment interfering behaviours (Howells \&Tennant, 2010) which impact and impede on engagement and ultimately treatment efficacy. The Westgate Unit uses a permutation of the physical environment, regime, and responsive psychological intervention in order to manage the critical impact that treatment inferring behaviours have on engagement and risk reduction (Bennett, 2015; Wood, 2015). It is unknown at present however, to what degree clinical disorder, and co-morbidity of personality disorder and clinical disorder exists within this specific population. Given that some domains of clinical disorder are mirrored within specific personality disorder traits, some co-morbidity would be expected. Therefore, broadening understanding would facilitate responsivity management and the allocation of appropriate assessment and/or treatment for this specific group of high-risk offenders. Thus this study was exploratory in nature with an aim to examine the prevalence of clinical disorders and the extent to which personality disorders, and clinical disorders co-exist within a sample of male offenders who have met criteria for a high-risk, high need, personality disorder treatment services.

\section{Method}

\section{Sample}

Participants were male personality disordered offenders $(n=115)$ who had met criteria for prisonbased, high secure personality disorder services and were located on a treatment unit specifically designed for this client group. The sample represents all available male participants who were admitted to the treatment unit and went on to meet criteria since it opened in 2004 and up until the point of data collection in 2015. Participants included within the research sample had all provided informed consent for their assessment data to be used for research purposes. 


\section{Procedure}

This was a retrospective study where participants' demographic and assessment data was selected for the purpose of the study. Participants had been assessed by a multidisciplinary team of qualified professionals upon admission with a standardised assessment procedure. Axis I disorders were assessed using the Structured Clinical Interview for DSM-IV for Axis I Disorders (SCID-I; First et al., 1997). These were administered by either a qualified mental health nurse or a qualified psychiatrist from the multidisciplinary team. Axis II disorders were assessed using the International Personality Disorder Examination (IPDE; Loranger, 1999). These were administered by a team of trained clinical staff (including trainee psychologists and therapists) led by a forensic psychologist practitioner whose role was to provide clinical oversight and supervision of the assessment process. For the purposes of the present study, diagnoses of personality disorder were coded into two categories: absent or present (probable diagnoses were recoded into present). The clinical assessment procedures for both Axis I and Axis II diagnoses was based upon interviews and a review of file records consisting of criminal history records and family background data. In addition, Axis I and Axis II diagnoses are completed independent of one another for all prisoners.

\section{Analysis}

The analytical strategy included the completion of Chi-squared analysis to determine the difference in frequency distribution between Axis I and Axis II diagnosis. Additionally, odds ratios from $2 \times 2$ (presence vs absence of diagnosis) tables were calculated. The chi-square statistic was used to determine the significance of each odds ratio. To reduce the risk of chance capitalisation, Bonferroni correction was used for the chi-square tests and a significance criterion of $p<0.05$ was used throughout.

\section{Results}

A review of the demographic characteristics of the sample showed that the majority of the sample were white in ethnicity (95\%) with the remaining $5 \%$ comprising participants of black, Asian or of mixed race. The mean participant age on admission was 35.58 years $(S D=14.38)$. In terms of marital status, $86 \%$ of the sample were single on admission to prison, followed by married/cohabiting $(6 \%)$, divorced/separated $(6 \%)$, widowed (1\%) with data not recorded for $1 \%$ of the sample. The majority of participants $(62 \%)$ had been convicted with a life sentence, followed by indeterminate for public protection (IPP) sentences (20\%) and determinate sentences (18\%). Offence specific characteristics highlighted that $62 \%$ of the sample had an index offence of physical violence and $38 \%$ of the sample had a sexually violent index offence.

Axis II and Axis I disorders

The prevalence of personality disorder in the sample was large, with $93 \%$ of the participants receiving at least one Axis II diagnosis. Eight participants were diagnosed with no personality disorders but had met criteria for the treatment service based on psychopathy level. Co-morbidity of Axis II disorders was more common than single diagnosis: of the 107 offenders given a personality disorder diagnosis, $94 \%$ received multiple diagnoses. The mean number of diagnosed personality disorder diagnoses was 2.67. The most frequently diagnosed personality disorder was antisocial $(n=104)$, followed by borderline $(n=70)$, and paranoid $(n=38)$. Most personality disorders were found in Cluster B disorders. The co-occurrence of individual and clustered Axis II disorders is displayed in Table 1.

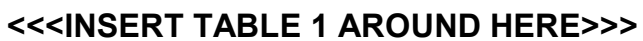


The prevalence of Axis I disorders was also high with $87 \%$ of the sample having at least one Axis I diagnosis. The most frequently diagnosed category was substance use disorders $(n=92,80 \%)$, followed by anxiety disorders $(n=54,47 \%)$ and mood disorders $(n=30,26 \%)$.

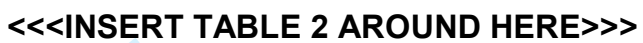 \\ Categorical overlap of Axis I and II disorders}

As evident in tables 1 and 2, several of the diagnostic categories have low base rates and diagnostic categories with fewer than ten cases were omitted from the analysis (Hart \& Hare, 1989). This resulted in five categories being omitted including two Axis I (somatoform and adjustment clinical disorders) and three Axis II (schizotypal, dependent and obsessive-compulsive personality disorders). Nevertheless, the omitted personality diagnoses were captured within both Cluster A and Cluster $C$ diagnostic categories. Due to Cluster B personality disorders having the highest level of diagnosis, there were not sufficient participants without a Cluster B personality disorder diagnosis to explore relationships between Cluster $\mathrm{B}$ personality disorder diagnoses and clinical disorder categories. Specific Cluster B personality disorder diagnoses (including antisocial and borderline personality disorders) were, however, explored within other analyses (see Table 3). Levels of co-morbidity between cluster diagnoses and clinical disorders were as follows. A significant positive trend was found between Cluster $A$ and mood disorder $\left(\chi^{2}(1)=4.191, p=.041\right)$ and between Cluster $A$ and schizophrenia/other psychotic disorder $\left(\chi^{2}(1)=4.75, p=.029\right)$ with odds ratios of 0.42 and 0.29 respectively.

\section{<<<INSERT TABLE 3 AROUND HERE >>}

$93(81 \%)$ of participants had diagnoses within both Axis I and Axis II disorders. With regards to the overlap between Axis II and clinical disorder category, a significant trend was found between paranoid personality disorder and mood disorder $\left(\chi^{2}(1)=7.552, p=.006\right)$ and between borderline personality disorder and mood disorder $\left(\chi^{2}(1)=9.208, p=.002\right)$ with odds ratios of 0.31 and 0.21 respectively.

With regards to Axis II and individual clinical disorders, significant trends were found between paranoid personality disorder and major depressive disorder $\left(\chi^{2}(1)=3.873, p=.049\right)$, hallucinogen abuse $\left(\chi^{2}(1)=10.062, p=.002\right)$ and specific phobia $\left(\chi^{2}(1)=4.184, p=.041\right)$. Schizoid personality disorder was significantly related to alcohol abuse $\left(\chi^{2}(1)=3.914, p=.048\right)$. Borderline personality disorder was significantly related to major depressive disorder $\left(\chi^{2}(1)=5.598, p=.018\right)$ and dysthymic disorder $\left(\chi^{2}(1)=4.842, p=.028\right)$. Narcissistic personality disorder was significantly related to alcohol abuse $\left(\chi^{2}(1)=3.977, p=.046\right)$, and avoidant personality disorder was significantly related to major depressive disorder $\left(\chi^{2}(1)=4.398, p=.036\right)$. Similar to previous results, relatively low odds ratios were calculated for these relationships (see Table 5). Most notable, however, was a higher odds ratio for the association between narcissistic personality disorder and alcohol abuse (2.57).

\section{<<<INSERT TABLES 4 \& 5 AROUND HERE $\gg>$}

\section{Discussion}

A high prevalence of lifetime DSM-IV Axis I psychiatric morbidity was found, with $87 \%$ of the sample meeting criteria for at least one Axis I disorder. This supports similarly high prevalence within 
personality disordered samples within high-security hospital settings (94\%; Blackburn et al., 2003). This high prevalence highlights the clinical complexity of this population and the importance of accurate clinical assessment to ensure that these distinct disorders are treated appropriately. This would ensure that treatment resources are allocated effectively and maximise risk reduction, ensuring that an efficient and successful treatment model is implemented. The finding that substance misuse was the most prevalent type of disorder is consistent with other studies with forensic participants (Coid et al., 2009), with research in high-secure hospitals highlighting depression as the most prevalent Axis I diagnosis, followed by alcoholism and substance misuse disorders (Coid, 2003). This finding supports the need for substance misuse interventions within personality disorder treatment services.

The high percentage of offenders with at least one Axis II diagnosis (93\%) was expected given the inclusion criterion for the treatment service where this study was based. In comparison to the general prison population (Coid et al., 2009) this figure is $28 \%$ higher. Antisocial personality disorder was the most prevalent personality disorder in the current sample $(90.43 \%)$. This is markedly higher than levels of antisocial personality disorder found in samples of males and females in high secure hospital samples (55\%; Coid, 2003) and the general prison population (50\%, Coid et al., 2009). This is of interest in terms of the current study given the associations between features relevant to this study's sample (namely antisocial personality disorder and psychopathy) and risk of (particularly violent) offending (Skilling et al., 2002).

The co-morbidity between Axis I and Axis II disorders was high, with $80.87 \%$ of the sample having diagnoses within both Axis I and Axis II disorders. Cluster A personality disorder was strongly associated with mood disorder and schizophrenia/other psychotic disorders. Further analysis illustrated that mood disorder was significantly related to paranoid (Cluster A) and borderline (Cluster B) personality disorders. Additionally, paranoid personality disorder and major depressive disorder were considered to have comorbidity. It has been suggested that paranoid personality disorder and mood disorders are significantly related within prison samples (Oldham et al., 1995; Coid et al., 2009); although this finding has not been replicated within high-security hospital samples (Coid, 2003). Borderline personality disorder and depression have been considered to be associated within general forensic, as well as high-security hospital settings (Coid, 2003; Coid et al., 2009), providing evidence to suggest that this was the finding most consistent with the literature base. A diagnosis of Cluster $\mathrm{C}$ personality disorder (which accounted for $23 \%$ of the sample) was not considered to be significantly linked to any Axis I disorders. However, within this cluster, avoidant personality disorder was positively linked to a diagnosis of major depressive disorder. This mirrors findings from a high security hospital sample (Coid, 2003); yet the same finding was not identified within the general prison population (Coid et al., 2009). These findings emphasise the clinical importance of personality disorder services being guided by research exploring their specific population - either prison or hospital - and research from general prison populations should be used tentatively. This further supports research and evaluation strategies within the Offender Personality Disorder Pathway.

No association was found between substance use disorder and personality disorder diagnosis within the present study. This was unexpected given that substance use is assessed within diagnoses of both antisocial and borderline personality disorders. This is inconsistent with existing research (e.g. Coid et al., 2009) that identified alcohol misuse disorder as the most co-occurring Axis I disorder with Axis II disorders within a general prison-based population. It is probable that this finding is due to substance use disorder being highly prevalent across this sample and personality disorder diagnosis could have been unable to differentiate between those diagnosed with a substance misuse disorder and those who are not. Further research could inform our understanding of 'what works' within substance use treatment provided for this population. The 'complex personality disorder' criterion for the unit where the study took place considers psychopathy as well as a diagnosis of personality disorder. Given this, it may be that one aspect of psychopathy (which relates to a "chronically 
unstable, antisocial and socially deviant lifestyle') may be more closely linked with substance misuse disorder than personality disorder.

Interestingly, anxiety disorders were not considered to hold comorbidity with any personality disorder within the current study. A more varying range of evidence supports the presence of co-morbidity between anxiety disorder and various personality disorders across the literature base. This includes: narcissistic (Coid et al., 2009; Links and Eynan, 2013); schizotypal (Coid et al., 2009; Blackburn et al., 2003); obsessive-compulsive (Coid et al., 2009); avoidant (Coid et al., 2009; Links and Eynan, 2013); antisocial (Links and Eynan, 2013; Hodgins et al., 2010); borderline, dependant (Links and Eynan, 2013); and paranoid personality disorders (Blackburn et al., 2003). This suggests there is varying evidence for the presence (and absence) of co-morbidity between Axis II and anxiety related Axis I disorders across samples.

When exploring specific clinical disorders, significant relationships were found between paranoid personality disorder and specific phobia, as well as hallucinogen abuse; schizoid personality disorder and alcohol abuse; borderline personality disorder and major depressive disorder and dysthymic disorder; narcissistic personality disorder and alcohol abuse; and avoidant personality disorder and major depressive disorder. This supports the need to treat clinical disorders in order to manage treatment interfering behaviours, thus maximising treatment engagement.

\section{Limitations}

Several methodological limitations are present within this study. Firstly, due to analysing secondary data collected over a lengthy period of time, no examination of inter-reliability could be carried out. Secondly, the study relied on data from one clinical service in the UK and consequently is limited by a potential lack of representativeness. Thirdly, due to the lower prevalence of some personality disorder and clinical disorder diagnoses within the sample, not all diagnoses could be explored restricting a comprehensive examination of potential interactions between multiple diagnoses. Additionally, the study explored categorical data in that a diagnosis of personality disorder or clinical disorder was considered to either be present or absent. Exploring the severity of personality and clinical disorders could develop findings from the current study further by using personality disorder dimensional scores as well as the severity of clinical disorders. The research strategy at the Westgate Unit intends to analyse data in order to contribute to ongoing evaluation. High psychopathy levels is a feature of this sample and to the best of our knowledge, no published study has examined the association between psychopathy, Axis I and Axis II disorders, thus warranting further exploration to develop further clinical insight into this specific population.

\section{Conclusions}

Despite the limitations, this study has provided insight into the prevalence of clinical disorders and the extent to which personality disorders, and clinical disorders co-exist within a sample of high-risk personality disordered offenders. Advancing this understanding is valuable given the association between clinical disorder, personality disorder and risk of re-offending (Skilling et al., 2002). It is hoped that further research can continue to build upon the current findings in order to extend this understanding further within this specific population. Thus, in part this study indicates that certain Axis II disorders may increase the risk of lifetime Axis I disorders and as such the findings must be applied to practice. This highlights the need to consider co-occurring diagnoses within treatment and risk management planning given the impact this can have on the treatment of personality and/or clinical disorder (Tyrer et al., 1997).

\section{Implications for practice}


- The prevalence of clinical disorders accentuates the importance of personality disorder treatment services providing therapy to address the clinical disorder symptoms that can interfere with personality disorder and risk focused treatment.

- Accurate assessment of personality disorder and clinical disorder is likely to reduce the risk of misdiagnosis and ensure the most appropriate treatment options are employed for both disorders.

- The prevalence of comorbidity within the sample emphasises the need for multidisciplinary staff working with this population to be suitability equipped to assess, treat and manage both types of disorder. Specifically, by seeing personality disorder and clinical disorders as distinct disorders that both require accurate and effective assessment and treatment within this challenging population.

- Given the introduction of the Offender Personality Disorder Pathway (Joseph \& Benefield, 2012) and its continuing development across settings and security levels, these implications could inform wider services within the pathway and consideration for resources allocation for treatment. Specifically, these implications could inform stakeholders' decision-making relating to both treatment provision and priorities.

Acknowledgement: The authors would like to acknowledge the contribution of all past and present members of the Mental Health and Psychology teams who have collected the data used in this study from the Westgate Personality Disorder Treatment Service opening to date. 
Table 1: Co-occurrence of Axis II disorder within the sample $(n=115)$

\begin{tabular}{ll}
\hline Axis II Cluster & $\begin{array}{l}\text { Categorical } \\
\text { Diagnosis }\end{array}$ \\
\hline Cluster A & $\mathrm{n}(\%)$ \\
Cluster B & $47(40.87)$ \\
Cluster C & $108(93.91)$ \\
\hline Axis II Disorder & $27(23.48)$ \\
\hline Paranoid & $\mathrm{n}(\%)$ \\
Schizoid & $38(33.04)$ \\
Schizotypal & $17(14.78)$ \\
Antisocial & $7(6.09)$ \\
Borderline & $104(90.43)$ \\
Histrionic & $70(60.87)$ \\
Narcissistic & $12(10.43)$ \\
Avoidant & $32(27.83)$ \\
Dependant & $23(20.00)$ \\
Obsessive-compulsive & $4(3.48)$ \\
\hline
\end{tabular}

Table 2: Co-occurrence of Axis I disorder within the sample $(n=115)$

\begin{tabular}{ll}
\hline Axis I Disorder Category & $\begin{array}{l}\text { Categorical } \\
\text { Diagnoses }\end{array}$ \\
\hline & $\mathrm{n}(\%)$ \\
\hline Mood & $30(26.09)$ \\
Schizophrenia and other Psychotic & $15(13.04)$ \\
Substance use & $92(80.00)$ \\
Anxiety & $54(46.96)$ \\
Somatoform & $4(3.48)$ \\
Adjustment & $2(1.74)$ \\
\hline
\end{tabular}


Table 3: Associations between Personality Disorder Clusters and Clinical Disorder Categories ( $n=115)$

\begin{tabular}{llllll}
\hline $\begin{array}{l}\text { Personality } \\
\text { Disorder } \\
\text { (Cluster) }\end{array}$ & Co-morbid Clinical Disorder Category & $\chi^{2}$ value & $\mathrm{df}$ & $\mathrm{p}$ & $\begin{array}{l}\text { Odds } \\
\text { ratio }\end{array}$ \\
\hline Cluster A & Mood & & & & \\
& Schizophrenia and other Psychotic & 4.750 & 1 & $.041^{*}$ & 0.42 \\
& Substance use & 0.229 & 1 & $.629^{*}$ & 0.29 \\
& Anxiety & 1.788 & 1 & .181 & 0.60 \\
Cluster C & Mood & 2.668 & 1 & .102 & 0.46 \\
& Schizophrenia and other Psychotic & 1.134 & 1 & .287 & 0.53 \\
& Substance use & 1.252 & 1 & .263 & 0.48 \\
& Anxiety & 0.038 & 1 & .846 & 1.09 \\
\hline
\end{tabular}

${ }^{*} p<.05$ 
Table 4: Associations between Personality Disorder Diagnoses and Clinical Disorder Categories $(n=115)$

\begin{tabular}{|c|c|c|c|c|c|}
\hline $\begin{array}{l}\text { Personality } \\
\text { Disorder } \\
\text { Diagnosis }\end{array}$ & Co-morbid Clinical Disorder Category & $\chi^{2}$ value & $\mathrm{df}$ & $\mathrm{p}$ & $\begin{array}{l}\text { Odd } \\
\text { ratio }\end{array}$ \\
\hline \multirow[t]{4}{*}{ Paranoid } & Mood & 7.552 & 1 & $.006^{*}$ & 0.31 \\
\hline & Schizophrenia and other Psychotic & 3.210 & 1 & .073 & 0.38 \\
\hline & Substance use & 0.409 & 1 & .522 & 0.71 \\
\hline & Anxiety & 2.306 & 1 & .129 & 0.55 \\
\hline \multirow[t]{4}{*}{ Schizoid } & Mood & 0.068 & 1 & .795 & 1.17 \\
\hline & Schizophrenia and other Psychotic & 0.029 & 1 & .865 & 1.15 \\
\hline & Substance use & 0.250 & 1 & .617 & 1.37 \\
\hline & Anxiety & 1.256 & 1 & .262 & 1.83 \\
\hline \multirow[t]{4}{*}{ Antisocial } & Mood & 0.365 & 1 & .546 & 1.48 \\
\hline & Schizophrenia and other Psychotic & 2.010 & 1 & .156 & 0.23 \\
\hline & Substance use & 0.053 & 1 & .819 & 1.20 \\
\hline & Anxiety & 0.025 & 1 & .873 & 1.10 \\
\hline \multirow[t]{4}{*}{ Borderline } & Mood & 9.208 & 1 & $.002^{* *}$ & 0.21 \\
\hline & Schizophrenia and other Psychotic & 1.278 & 1 & .258 & 0.50 \\
\hline & Substance use & 0.759 & 1 & .384 & 1.55 \\
\hline & Anxiety & 1.307 & 1 & .253 & 0.65 \\
\hline \multirow[t]{4}{*}{ Histrionic } & Mood & 1.687 & 1 & .094 & 0.45 \\
\hline & Schizophrenia and other Psychotic & 2.010 & 1 & .156 & 4.38 \\
\hline & Substance use & 0.053 & 1 & .819 & 0.83 \\
\hline & Anxiety & 1.906 & 1 & .167 & 0.42 \\
\hline \multirow[t]{4}{*}{ Narcissistic } & Mood & 1.944 & 1 & .163 & 0.53 \\
\hline & Schizophrenia and other Psychotic & 0.424 & 1 & .515 & 1.56 \\
\hline & Substance use & 2.690 & 1 & .101 & 2.23 \\
\hline & Anxiety & 0.836 & 1 & .360 & 0.68 \\
\hline \multirow[t]{4}{*}{ Avoidant } & Mood & 3.100 & 1 & .078 & 0.42 \\
\hline & Schizophrenia and other Psychotic & 0.633 & 1 & .426 & 0.60 \\
\hline & Substance use & 0.531 & 1 & .466 & 0.62 \\
\hline & Anxiety & 0.052 & 1 & .820 & 0.90 \\
\hline
\end{tabular}

${ }^{*} p<.01{ }^{* *} p<.005$

Table 5: Significant Associations between Personality Disorder Diagnoses and Clinical Disorders $(n=115)$

\begin{tabular}{llllll}
\hline $\begin{array}{l}\text { Personality } \\
\text { Disorder } \\
\text { Diagnosis }\end{array}$ & Co-morbid Clinical Disorder & $\chi^{2}$ value & df & $p$ & $\begin{array}{l}\text { Odds } \\
\text { ratio }\end{array}$ \\
\hline Paranoid & Major depressive disorder & 3.873 & 1 & $.049^{*}$ & 0.31 \\
& Specific phobia & 4.184 & 1 & $.041^{*}$ & 0.32 \\
& Hallucinogen abuse & 10.062 & 1 & $.002^{* *}$ & 0.27 \\
Schizoid & Alcohol abuse & 3.914 & 1 & $.048^{*}$ & 0.35 \\
Borderline & Major depressive disorder & 5.598 & 1 & $.018^{*}$ & 0.11 \\
& Dysthymic disorder & 4.842 & 1 & $.028^{*}$ & 0.13 \\
Narcissistic & Alcohol abuse & 3.977 & 1 & $.046^{*}$ & 2.57 \\
Avoidant & Major depressive disorder & 4.398 & 1 & $.036^{*}$ & 0.28 \\
\hline
\end{tabular}

${ }^{*} p<.05{ }^{* *} p<.005$ 


\section{References}

Bennett, A. L. (2015), "The Westgate service and related referral, assessment, and treatment processes", International journal of offender therapy and comparative criminology, Vol. 59, pp. 15801604.

Blackburn, R. (2000), "Treatment or incapacitation? Implications of research on personality disorders for the management of dangerous offenders", Legal and Criminological Psychology, Vol. 5, No. 1, pp. $1-22$.

Blackburn, R., Logan, C., Donnelly, J., and Renwick, S. (2003), "Personality disorders, psychopathy and other mental disorders: Co-morbidity among patients at English and Scottish high-security hospitals", The Journal of Forensic Psychiatry and Psychology, Vol. 14 No. 1, pp. 111-137.

Coid, J. W. (2003), "The co-morbidity of personality disorders and lifetime clinical syndromes in dangerous offenders", The Journal of Forensic Psychiatry and Psychology, Vol. 14, No. 2, pp. 341366.

Coid, J. W., Moran, P., Bebbington, P., Brugha, T., Jenkins, R., Farrell, M., Singleton, N. and Ullrich, S. (2009), "The co-morbidity of personality disorder and clinical syndromes in prisoners", Criminal Behaviour and Health, Vol. 19, No. 5, pp. 321-333.

Department of Health, National Offender Management Service Offender Personality Disorder Team. (2011), "Response to the Offender Personality Disorder Consultation", London: Department of Health.

First, M. B., Spitzer, R. L., Gibbon, M., and Williams, J. B. (1997), User's guide for the Structured clinical interview for DSM-IV axis I disorders SCID-I: clinician version. American Psychiatric Pub.

Hart, S. D., and Hare, R. D. (1989), "Discriminant validity of the Psychopathy Checklist in a forensic psychiatric population", Psychological Assessment: A Journal of Consulting and Clinical Psychology, Vol. 1, No. 3, pp. 211.

Hodgins, S., De Brito, S. A., Chhabra, P. and Côté, G. (2010), Anxiety disorders among offenders with antisocial personality disorders: a distinct subtype?, Canadian journal of psychiatry. Revue canadienne de psychiatrie, Vol. 55, No. 12, pp. 784-791.

Howells, K., and Tennant, A. (2010), "Ready or not, they are coming: Dangerous and severe personality disorder and treatment engagement", in Tennant, A. and Howells, K. (Eds.), Using time, not doing time: Practitioner perspectives on personality disorder and risk, Wiley, Chichester, UK, pp. 33-44.

Joseph, N., and Benefield, N. (2012), "A joint offender personality disorder pathway strategy: An outline summary", Criminal Behaviour and Mental Health, Vol. 22, No. 3, pp. 210-217.

Links, P. S., and Eynan, R. (2013), "The relationship between personality disorders and axis I psychopathology: deconstructing comorbidity”, Annual review of clinical psychology, Vol. 9, pp. 529554.

Loranger, A. W. (1999), International Personality Disorder Examination Manual: DSM-IV Module. Washington DC: American Psychiatric Press. 
Oldham, J. M., Skodol, A. E., Kellman, H. D., Hyler, S. E., Doidge, N., Rosnick, L., and Gallaher, P. E. (1995), "Comorbidity of axis I and axis II disorders", American Journal of Psychiatry, Vol. 152, No. 4, pp. 571-578.

Saradjian, J., Murphy, N., and McVey, D. (2013), "Delivering effective therapeutic interventions for men with severe personality disorder within a high secure prison", Psychology, Crime and Law, Vol. 19, Nos. 5-6, pp. 433-447.

Skilling, T. A., Harris, G. T., Rice, M. E., and Quinsey, V. L. (2002), "Identifying persistently antisocial offenders using the Hare Psychopathy Checklist and DSM antisocial personality disorder criteria. Psychological Assessment", Vol. 14, No. 1, p. 27.

Skodol, A. E., Grilo, C. M., Keyes, K. M., Geier, T., Grant, B. F. and Hasin, D. S. (2011), "Relationship of personality disorders to the course of major depressive disorder in a nationally representative sample", American Journal of Psychiatry, Vol. 168, No. 3, pp/ 257-264.

Tyrer P, Gunderson J, Lyons M, and Tohen M. (1997), "Special feature: Extent of comorbidity between mental state and personality disorders", Journal of Personality Disorders, Vol. 11, pp. 242259.

Wood F. (2015), "Working with Personality Disordered Offenders; responsivity issues and management strategies". Prison Service Journal, Vol. 218, pp. 24-30.

Zanarini, M. C., Frankenburg, F. R., Dubo, E. D., Sickel, A. E., Trikha, A., Levin, A., and Reynolds, V. (1998), "Axis II comorbidity of borderline personality disorder", Comprehensive Psychiatry, Vol. 39, No. 5, pp. 296-302. 